\title{
Application of the ACOSOG Z0011 Criteria to Chinese Patients with Breast Cancer: A Prospective Study
}

\section{Yuan Peng}

Peking University People's Hospital

Miao Liu

Peking University People's Hospital

\section{Fuzhong Tong}

Peking University People's Hospital

\section{Yingming Cao}

Peking University People's Hospital

\section{Peng Liu}

Peking University People's Hospital

\section{Bo Zhou}

Peking University People's Hospital

Hongjun Liu

Peking University People's Hospital

\section{Lin Cheng}

Peking University People's Hospital

\section{Jiajia Guo}

Peking University People's Hospital

\section{Fei Xie}

Peking University People's Hospital

Houpu Yang

Peking University People's Hospital

\section{Siyuan Wang}

Peking University People's Hospital

\section{Chaobin Wang}

Peking University People's Hospital

Shu Wang ( $\nabla$ shuwang@pkuph.edu.cn )

Peking University People's Hospital https://orcid.org/0000-0002-8651-4795 
Keywords: Breast cancer, Sentinel lymph node, ACOSOG Z0011, Chinses

Posted Date: September 14th, 2020

DOI: https://doi.org/10.21203/rs.3.rs-74141/v1

License: (c) (1) This work is licensed under a Creative Commons Attribution 4.0 International License. Read Full License

Version of Record: A version of this preprint was published at World Journal of Surgical Oncology on April 20th, 2021. See the published version at https://doi.org/10.1186/s12957-021-02242-1. 
Title page

Title

Application of the ACOSOG Z0011 criteria to Chinese patients with breast cancer: A prospective study

Authors

1. Yuan Peng, Breast Center, Peking University People's Hospital, Beijing, China

2. Miao Liu, Breast Center, Peking University People's Hospital, Beijing, China

3. Fuzhong Tong, Breast Center, Peking University People's Hospital, Beijing, China

4. Yingming Cao, Breast Center, Peking University People's Hospital, Beijing, China

5. Liu Peng, Breast Center, Peking University People's Hospital, Beijing, China

6. Bo Zhou, Breast Center, Peking University People's Hospital, Beijing, China

7. Hongjun Liu, Breast Center, Peking University People's Hospital, Beijing, China

8. Lin Cheng, Breast Center, Peking University People's Hospital, Beijing, China

9. Jiajia Guo, Breast Center, Peking University People's Hospital, Beijing, China

10. Fei Xie, Breast Center, Peking University People's Hospital, Beijing, China

11. Houpu Yang, Breast Center, Peking University People's Hospital, Beijing, China

12. Siyuan Wang, Breast Center, Peking University People's Hospital, Beijing, China

13. Chaobin Wang, Breast Center, Peking University People's Hospital, Beijing, China

14. *Shu Wang (corresponding author), Breast Center, Peking University People's Hospital, Beijing, China 
Abstract

Background: Although the ACOSOG Z0011 study showed axillary lymph node dissection (ALND) could be avoided in a specific population of sentinel lymph node positive patients, it's not widely accepted by Chinese surgeons. We conducted a prospective single-arm study to confirm whether or not the results of Z0011 are applicable to Chinese patients.

Methods: Patients conforming to the Z0011 criteria were prospectively enrolled at the Peking University People's Hospital Breast Center from November 2014 to June 2019. Clinicopathological features of the study group were compared with the Z0011 study. Lymphedema after surgery, incidence of local-regional recurrence, and survival were analyzed.

Results: One hundred forty-two patients who met Z0011 eligibility criteria were enrolled in this study; 115 had sentinel lymph node biopsy (SLNB) alone. When comparing with the Z0011 trial, younger patients were included (median age, 52 [2682] years vs 54 [25-90] years; $\mathrm{P}=0.03$ ). Among clinical $\mathrm{T}$ stage, tumor histology, hormone status, lymphovascular invasion, and the number of positive sentinel lymph nodes (SLNs), no statistically significant differences were observed. More patients received adjuvant chemotherapy and endocrine therapy $(90.85 \%$ vs $58.0 \%$ and $80.99 \%$ vs $46.6 \%$ respectively, $\mathrm{P}<.001)$. A similar percentage of patients received radiotherapy, but more nodal radiotherapy procedures were carried out in our study (54.5\% vs $16.9 \%)$. After median follow-up of 29 months, only 1 patient $(0.9 \%)$ had ipsilateral breast tumor recurrence and no regional recurrence occurred.

Conclusion: Our study showed that it is achievable to avoid ALND in patients eligible for Z0011 in China.

Trial registration: ClinicalTrials Registered Number: NCT03606616. Registered 31 July 2018-Retrospectiverly registered, https://www.clinicaltrials.gov/ct2/show/NCT03606616?term=Wang+shu\&draw=4\&ra $\mathrm{nk}=21$.

Keywords

Breast cancer, Sentinel lymph node, ACOSOG Z0011, Chinses 


\section{Background}

The American College of Surgeons Oncology Group (ACOSOG) Z0011 trial is the largest prospective, randomized controlled study comparing local control rates and overall survival rates between axillary lymph node dissection (ALND) and sentinel lymph node biopsy (SLNB) groups in patients with positive SLNs. The results of the trial showed that local-regional control and overall survival for patients receiving SLNB alone was not inferior to receiving ALND in 10 years $[1,3]$. And the results of this study showed a major effect on the clinical practice of breast surgery; since 2012, the National Comprehensive Cancer Network guidelines have been continually changed to this day [2].

After the Z0011 trial, America, Australia, Europe, Japan, and other regions have verified the results in their own populations [4-10]. Although several studies using different database revealed that ALND could be omitted based on the Z0011 strategy [11-12], the attitudes of surgeons are controversial. According to a survey in 2018 in America, $49 \%$ of surgeons would recommend ALND for 1 SLN metastasis and $63 \%$ would recommend ALND for 2 SLN metastases [13]. In China, the attitude is more negative, only $16.6 \%$ of hospitals accepted the conclusions of the Z0011 study [14].

In 2014, we led a retrospective analysis and found that the clinicopathological factors were not statistically different between the eligible group in China and the Z0011 cohort. These findings laid the foundation for omitting ALND in Chinese patients according to the Z0011 criteria [15]. However, no prospective clinical trial result has been reported in the Chinese population. The current study is the first to assess whether the Z0011 criteria to avoid ALND after positive SLNs findings are applicable to Chinese patients with breast cancer.

\section{Methods}

Beginning in November 2014, we adapted the results of the Z0011 trial to the management of patients with breast cancer at Peking University People's Hospital (PKUPH) Breast Center. This was a prospective, single-arm study. From November 2014 to June 2019, patients with invasive breast cancer were enrolled if they met the following Z0011 trial criteria: (1) diagnosed with clinical stage T1-2N0 cancer and (2) previously underwent breast-conserving surgery with planned whole-breast irradiation. A waiver of ethic approval was issued by PKUPH's review board and written informed consent was obtained from all the patients. The trial was registered as NCT03606616 at the following site: https://clinicaltrials.gov.

All patients received routine preoperative axillary nodal ultrasound imaging. Fineneedle biopsy was allowed for suspicious lymph nodes. If the aspiration cytology suggested malignancy, ALND was performed.

SLNs were detected using blue dye and indocyanine green. All blue staining or fluorescent-labeled lymph nodes were removed. Any patients with negative SLNs or isolated tumor cells within the SLNs were excluded from further analysis. ALND was performed if 3 or more positive nodes were detected or within nodes with gross extracapsular extension. Adjuvant treatment for each patient was based on national guidelines and physicians' choices. Whole-breast radiation therapy had to be included, 
in addition to other radiotherapy fields depending on treatment specified by the radiation oncologists.

We collected the clinical and pathological data, including the adjuvant therapies. Local-regional recurrence, distant metastasis, and survival were closely monitored. The presence of lymphedema was reported in one of 2 ways: (1) self-report by the patient or (2) physician diagnosis and use of the Breast Cancer and Lymphedema Symptom Experience Index (BCLE-SEI)

All data were analyzed using SPSS, version 20.0 statistical software. The clinicopathological features of the study group and the Z0011 trial SLNB cohort were compared. The characteristics of the eligible group and the Z0011 SLNB alone group were compared using chi-square and $t$ tests. A $P$ value $<.05$ was considered significant. The survival data were analyzed with descriptive statistics.

\section{Results}

In the current study, 828 patients with invasive breast cancer were enrolled from November 2014 to June 2019. The patient flow chart is shown in Figure 1. Six hundred eighty-six patients with (1) negative SLNs, (2) isolated tumor cells, (3) 3 or more positive SLNs, or (4) nodes with gross extracapsular extension were excluded from the study and underwent ALND. One hundred forty-two patients were eligible and therefore remained in the analysis. Twenty-seven patients otherwise eligible for SLNB alone underwent ALND as a result of either surgeon or patient preference.

Table 1 shows the baseline characteristics of the 142 eligible patients. The median age of the patients was 52 (range, 26-82) years old, and 112 (78.87\%) patients presented with clinical T1 tumors. Patients who were hormone receptor positive accounted for $82.39 \%$. In $76.76 \%$ of patients, only 1 metastatic lymph node was found among SLNs. Adjuvant chemotherapy was given to $129(90.85 \%)$ patients and adjuvant endocrine therapy to $115(80.99 \%)$. In the 27 patients who underwent ALND, 7 (25.93\%) had additional positive nodes (range, 1-9). Among 115 patients treated with SLNB alone, $101(87.8 \%)$ received radiotherapy. Detailed radiotherapy records were obtained for 99 patients. Of these, $54(54.55 \%)$ patients received breast and nodal radiotherapy (nodal radiotherapy included the level III axillary and supraclavicular nodes) and 23 (23.23\%) patients received high-tangent radiotherapy. The features of each group of patients are shown in Table 2.

The pathological and clinical characteristics of patients in the Z0011 trial SLNBalone arm were compared to our patients in the current study; these comparisons are shown in Table 3. Among clinical $\mathrm{T}$ stage, tumor histology, hormone status, lymphovascular invasion, and the number of positive SLNs, no statistically significant differences were observed. Our eligible patients were younger than those in the Z0011 trial, and most patients received chemotherapy and endocrine therapy $(P<.001)$.

No axillary recurrences have occurred in our study at a median follow-up of 29 months (range, 5-60 months). One patient had ipsilateral breast tumor recurrence 56 months after operation.

We administered a questionnaire using BCLE-SEI in all patients. Sixteen patients were at risk of lymphedema (symptom score, 2-8 points). The scores in the ALND 
group were higher than those in the SLN-alone group (Table 4). In patients from the SLN-alone group, $2(1.7 \%)$ were either self-reported cases of lymphedema or had a physician diagnosis of lymphedema. Three (11.1\%) patients who had ALND reported lymphedema.

\section{Discussion}

In recent years, the clinical practice of breast surgery has been greatly influenced by the ACOSOG Z0011 trial. In Europe and Australia, the rate of ALND decreased obviously after the Z0011 study published [16-17]. And in China, ALND has been the standard treatment for patients with positive SLNs until the guidelines of Breast Cancer Committee affiliated with the Chinese Anti-Cancer Association were revised in 2019 [18]. The reason why the Z0011 trial results have not been accepted by most Chinese surgeons in the past few years may be because of the lack of Chinese patients' own data. It is unknown if Chinese patients with breast cancer and patients from the West with breast cancer share clinical characteristics similar to those of the Z0011 trial. It is also unclear if results similar to those of the Z0011 trial can be achieved in a population of Chinese patients under the current adjuvant treatment pattern in China, especially the excellent local-regional control. In our study, we prospectively investigated whether or not the Z0011 criteria could feasibly be applied to Chinese patients.

In our prospective study, 142 patients met the ACOSOG Z0011 eligibility criteria and 115 patients no longer underwent ALND. Although our patients were a little bit younger than the patients of the Z0011 trial, the clinical T stage, tumor histology, hormone status, lymphovascular invasion, and the number of positive SLNs showed no remarkable difference between both studies. In our study, $25.94 \%$ (7 out of 27) patients had additional positive nodes after ALND, similar to previous studies [5-7, 15,19] (Table 5). Although a 1 out of 4 possibilities of non-SLN metastasis exists, we still achieved very good local-regional control and survival. Only 1 patient experienced ipsilateral breast recurrence, and no regional recurrence or death occurred. This result is consistent with that of the Z0011 trial, which showed that potential residual positive lymph nodes could be successfully controlled by radiotherapy and systemic therapies. Therefore, ALND can be performed to avoid SLN positivity in a large majority of patients following the Z0011 criteria. In our study, ALND was avoided in $73.25 \%$ (115 out of 157) of positive SLNs, similar to other retrospective and prospective reports [5,7,10,19-20].

There are differences between patients in the Z0011 trial and those eligible for our analysis. First, most studies used radioisotopes, blue dye, or in the case of Japan, indocyanine green and technetium tin colloids for SLNB [5-6, 8]. Our study used blue dye and indocyanine green. According to previous research reports, indocyanine green in conjunction with blue dye is an efficient method [21-22] to detect SLNs without affecting the results.

Second, we conducted a rigorous preoperative assessment. The Z0011 trial applied no specific requirements for preoperative axillary lymph node imaging assessment, unless the enlarged axillary lymph nodes were palpable, according to United States 
guidelines. However, in our opinion, imaging assessment of axillary lymph nodes before surgery may be useful. If a lymph node is found to be positive on ultrasoundguided fine-needle biopsy, a higher nodal burden is predicted than on a positive SLNB [19,23-24]. Under the European guidelines [25-26], in patients with or without palpable lymph nodes, axillary ultrasound is a routine diagnostic procedure. This is true according to the Chinese guidelines as well [18]. According to the guidelines in China, our study required the preoperative assessment of the axillary nodal status of all patients using ultrasound. If fine-needle aspiration cytology for suspicious lymph nodes was positive, then the patients were ineligible to enroll. However, in Morrow's study, the researchers excluded routine imaging of the axilla with ultrasound or magnetic resonance imaging [4]. In their opinion, even if the image guided aspiration was positive, there are still some patients with only 1 or 2 positive nodes who were able to avoid ALND; further, the local-regional control was unaffected. Moreover, in our previous study, we assumed that if only one abnormal lymph node is detected on ultrasound, then fine-needle biopsy could be omitted but not for multiple suspicious nodes [27]. Perhaps axillary ultrasound assessment and fine-needle aspiration are not necessary for all patients, but we cannot omit use of either today in China.

Third, in practice, more patients received regional nodal radiotherapy. In the Z0011 trial, among the patients with radiotherapy records, $52.6 \%$ were treated by hightangent radiotherapy, and $16.9 \%$ received treatment of the supraclavicular region [28]. In Morrow's study, $21 \%$ of the patients received breast and nodal irradiation, and 58\% received supine breast radiotherapy (this method allows patients to receive more axillary I/II radiotherapy than prone breast radiation therapy). Meanwhile, 23.2\% of our patients received high-tangent irradiation, and 54.5\% included treatment of the nodal radiotherapy (level III axillary and supraclavicular nodes). The radiotherapy field in several prospective studies is shown in Figure 2. The choice of irradiation field is directly related to the understanding of recurrence risks by radiation physicians. Patients with high risks of recurrence, including young age, larger tumor size, hormone receptor negative, and HER2 positive, were more likely to receive nodal RT in our study. The use of nodal RT increased with the number of positive SLNs; this is consistent with the Z0011 and Morrow study. The high-risk patients treated with heavier radiation have also been confirmed in a nomogram-based study [29]. It has been hypothesized that radiation oncologists, who could not be blinded to the surgical treatment of the patients nor to the pathological results after surgery, may have treated patients on the SLN-only arm with high-tangent radiotherapy to include a component of axillary level I/II and even three-field radiation more often than those in the ALND arm, particularly for patients at high risk. Even in America, nearly half of Z0011-eligible patients receive regional nodal irradiation from National Cancer Database study [30]. Close multidisciplinary teamwork between clinical oncologist and radiation physicians are needed to optimize the radiotherapy field. And the optimal radiation therapy for these patients still need further study.

Fourth, more patients in our study received chemotherapy and endocrine therapy compared with the Z0011 trial $(P<.001)$. Chemotherapy and endocrine therapy can improve the prognosis of patients with breast cancer, whether in terms of local- 
regional control or overall survival. Positive lymph nodes are one of the indications for adjuvant chemotherapy per the Chinese Society of Clinical Oncology guidelines. The use of prognostic multigene signatures such as Oncotype DX or MammaPrint may influence results in the future in China. We believe that reducing chemotherapy in some low risk patients will not affect the prognosis of these patients.

The use of SLNB alone resulted in fewer complications. In the Z0011 trial, lymphedema was reported by $13 \%$ of patients after ALND and $2 \%$ of patients after SLNB alone at 1 year. Lymphedema diagnosed by arm circumferences (defined as a 2 $\mathrm{cm}$ or greater postoperative increase in ipsilateral arm measurements compared with the contralateral arm) was $6 \%$ vs $11 \%$ in the 2 arms, respectively [31]. Lymphedema evaluation methods varied in different studies. In the IBCSG 23-01 trial, the treating physician reported edema; assessments were based on the National Cancer Institute Common Toxicity Criteria version 2. The incidence of lymphedema was $3 \%$ in the SLN group and $13 \%$ in the ALND group (median follow-up of 5 years) [32]. Our patients received more nodal RT compared with Z0011 trial, but did not see a significant increase in lymphedema. Lymphedema reported by patients or physicians was $11.1 \%$ after ALND and $1.7 \%$ after SLNB alone with a median follow-up of 29 months. In a trial comparing radiotherapy and ALND after SLNB, at 5 years, the lymphedema reported by arm circumference was 5\% in the radiotherapy arm and $13 \%$ in the ALND arm [33]. From the above data, even after receiving axillary radiotherapy after SLNB, the incidence of edema did not increase significantly, and the proportion of edema was significantly reduced compared to ALND. However, a longer follow-up period is required.

This study has several limitations. First, the median follow-up of 29 months was short, and the number of patients was insufficient to draw final conclusions about the incidence of local-regional recurrences. However, thus far, our results demonstrate an extremely low rate of local-regional recurrence in Chinese patients considering the diagnosis and treatment pattern today. Long-term follow-up is suggested to confirm the reliability of our data. Second, our results reflect a single-center experience and the adjuvant treatments are influenced by individual physician's preferences, such as the use of adjuvant chemotherapy and the irradiation field. The results of this trial should be confirmed by multicenter studies.

To our knowledge, this prospective study is the first to apply the ACOSOG Z0011 criteria to Chinese patients with early stage breast cancer. Our study demonstrates (1) a low risk of local-regional recurrence and (2) a good prognosis in patients with positive SLNs who were treated with SLNB alone. We believe that the results of our pilot study regarding the Chinese patient population will have a great effect on the clinical practice of Chinese surgeons in treating patients with breast cancer. 
List of abbreviations

ALND axillary lymph node dissection

SLNB sentinel lymph node biopsy

SLN sentinel lymph node

PKUPH Peking University People's Hospital

BCLE-SEI Breast Cancer and Lymphedema Symptom Experience Index

\section{Declarations}

Ethics approval and consent to participate

The study was approved by the Peking university People's hospital ethics committee.

Committee's reference number: 2018PHB152

Consent for publication

Not applicable

Availability of data and materials

The datasets used and/or analyzed during the current study are available from the corresponding author on reasonable request.

Competing interests

The authors declare that they have no competing interests.

Funding

This study was funded by Natural Science Foundation of Beijing Municipality (grant 7202212), and Capital Foundation of Medical Development (grant: A Multi-center Clinical Trial of Intra-operative Artificial Intelligence Aided Evaluation of Sentinel Lymph Nodes Using Dynamic Cellular Imaging Based on Optical Coherence Tomography).

Authors 'contributions

Yuan Peng, the acquisition, analysis, and interpretation of data, have drafted the work or substantively revised it.

Miao Liu, Fuzhong Tong, Yingming Cao, Liu Peng, Bo Zhou, Hongjun Liu, Lin Cheng, Jiajia Guo, Fei Xie, Houpu Yang, Siyuan Wang, Chaobin Wang, the acquisition of data.

Shu Wang, substantial contributions to the conception, design of the work, have drafted the work or substantively revised it.

All authors read and approved the final manuscript

Acknowledgements

Not applicable 


\section{References}

1. Giuliano AE, Hunt KK, Ballman KV, et al. Axillary dissection vs no axillary dissection in women with invasive breast cancer and sentinel node metastasis: a randomized clinical trial. JAMA.2011;305:569-75.

2. National Comprehensive Cancer Network (NCCN) Clinical practice guidelines in oncology: breast, version 1.2012. http://

www.nccn.org/professionals/physician_gls/pdf/ breast.pdf Accessed 30 Jun 2017.

3. Giuliano AE, Ballman KV, McCall L et al. Effect of axillary dissection vs no axillary dissection on 10-year overall survival among women with invasive breast cancer and sentinel node metastasis: the ACOSOG Z0011 (Alliance) randomized clinical trial. JAMA.2017.318:918-26.

4. Dengel, L. T. et al. Axillary dissection can be avoided in the majority of clinically node-negative patients undergoing breast conserving therapy. Ann Surg Oncol.2014,21:22-7.

5. Delpech, Y. et al. The exportability of the ACOSOG Z0011 criteria for omitting axillary lymph node dissection after positive sentinel lymph node biopsy findings: a multicenter study. Ann Surg Oncol.2013,20:2556-61.

6. Aigner, J. et al. Omission of axillary dissection according to ACOSOG Z0011: impact on adjuvant treatment recommendations. Ann Surg.2013,20:1538-44.

7. Ngui, N. K., Elder, E. E., Jayasinghe, U. W. \& French, J. Relevance of the American College of Surgeons Oncology Group Z0011 Trial to breast cancer in the Australian setting. ANZ J Surg.2013,83: 924-8.

8. Kittaka N, Tokui R, Hashimoto Y, et al. A prospective feasibility study applying the ACOSOG Z0011 criteria to Japanese patients with early breast cancer undergoing breast-conserving surgery. Int J Clin Oncol.2018,23:860-6.

9. Jiwoong Jung, Wonshik Han, Eun Sook Lee, et al. Retrospectively Validating the Results of the ACOSOG Z0011 Trial in a Large Asian Z0011-eligible Cohort. Breast Cancer Research and Treatment. 2019,175:203-15.

10. Monica Morrow, Kimberly J. Van Zee, Sujata Patil, et al. Axillary dissection and nodal irradiation can be avoided for most node-positive Z0011-eligible breast cancers: a prospective validation study of 793 patients. Ann Surg. 2017,266:457-62.

11. Jung J, Kim BH, Kim J, et al. Validating the ACOSOG Z0011 Trial Result: A Population-Based Study Using the SEER Database. Cancers (Basel). 2020,12:950.

12. Jina Lee, Jung Eun Choi, Sei Joong Kim, et al. Comparative Study between Sentinel Lymph Node Biopsy and Axillary Dissection in Patients with One or Two Lymph Node Metastases. J Breast Cancer.2018,21:306-14.

13. Morrow M, Jagsi R, McLeod M.C, et al. Surgeon Attitudes Toward the Omission of Axillary Dissection in Early Breast Cancer. JAMA Oncol.2018,4:1511-6.

14. Xin Zhang, Ying Wang. A Survey of Current Surgical Treatment of Early Stage Breast Cancer in China. Oncoscience.2018,5:239-47.

15. Miao Liu, Shu Wang, Shude Cui, et al. The feasibility of the ACOSOG Z0011

Criteria to Chinese Breast Cancer Patients: A Multicenter Study. Scientific

Reports.2015,5:15241.

16. Carlos A Garcia-Etienne, Robert E Mansel, Mariano Tomatis, et al. Trends in 
Axillary Lymph Node Dissection for Early-Stage Breast Cancer in Europe: Impact of Evidence on Practice. The Breast.2019,45:89-96.

17. Nicholas K Ngui, Kerry Hitos, T Michael D Hughes, et al. Effect of the American College of Surgeons Oncology Group Z0011 Trial on Axillary Management in Breast Cancer Patients in the Australian Setting. Breast J.2019,25:853-58.

18. Breast Cancer Committee of China Anti-Cancer Association. Guidelines in breast cancer diagnosis and treatment of China Anti-Cancer Association (version 2019).

Zhongguo Ai Zheng Za Zhi.2019,29:609-80.

19. Verheuvel NC, Voogd AC, Tjan-Heijnen VC, et al. Potential impact of application of Z0011 derived criteria to omit axillary lymph node dissection in node positive breast cancer patients. Eur J Surg Oncol. 2016,42:1162-8.

20. Yi M, Kuerer HM, Mittendorf EA, et al. Impact of the American college of surgeons oncology group Z0011 criteria applied to a contemporary patient population. J Am Coll Surg.2013,216:105-13.

21. Long Yuan, Xiaowei Qi, Yi Zhang, et al. Comparison of sentinel lymph node detection performances using blue dye in conjunction with indocyanine green or radioisotope in breast cancer patients: a prospective single-center randomized study. Cancer Biol Med. 2018,15:452-60.

22. Stephanie A Valente, Zahraa Al-Hilli, Diane M Radford et al. Near Infrared Fluorescent Lymph Node Mapping with Indocyanine Green in Breast Cancer Patients: A Prospective Trial. J Am Coll Surg.2019,228:672-78.

23. Boland MR, Ni Cearbhail R, Fitzpatrick K, et al. A Positive Node on UltrasoundGuided Fine Needle Aspiration Predicts Higher Nodal Burden Than a Positive Sentinel Lymph Node Biopsy in Breast Carcinoma. World J Surg. 2016,40:2157-62. 24. Liang Y, Chen X, Tong Y, et al. Higher Axillary Lymph Node Metastasis Burden in Breast Cancer Patients with Positive Preoperative Node Biopsy: May Not Be Appropriate to Receive Sentinel Lymph Node Biopsy in the post-ACOSOG Z0011 Trial Era. World J Surg Oncol.2019,17:37.

25. Senkus E, Kyriakides S, Penault-Llorca F, et al. Primary breast cancer: ESMO clinical practice guidelines for diagnosis, treatment and follow up. Ann Oncol. 2013,24 Suppl 6:vi7-23.

26. Harnett A, Smallwood J, Titshall V, et al. Diagnosis and treatment of early breast cancer, including locally advanced disease summary of NICE guidance.

BMJ.2009,338: b438.

27. Miao Liu, Yang Yang, Fei Xie, et al. Could axillary clearance be avoided in clinically node-negative breast cancer patients with positive nodes diagnosed by ultrasound guided biopsy in the post-ACOSOG Z0011ear? PLoS One.2019,14:e210437.

28. Jagsi R, Chadha M, Moni J et al. Radiation field design in the ACOSOG Z0011 (Alliance) trial. J Clin Oncol.2014,32:3600-6.

29. Matthew S. Katz, Linda McCall, Karla Ballman, et al. Nomogram-based estimate of axillary nodal involvement in ACOSOG Z0011 (Alliance): validation and association with radiation protocol variations. Breast Cancer Research and Treatment. 2020,180:429-36. 
30. Benjamin M Raber, Heather Lin, Yu Shen, et al. Trends in Regional Nodal Management of Breast Cancer Patients With Low Nodal Burden. Ann Surg Oncol.2019,26:4346-54.

31. Anthony Lucci, Linda Mackie McCall, Peter D. Beitsch, et al. Surgical Complications Associated With Sentinel Lymph Node Dissection (SLND) Plus Axillary Lymph Node Dissection Compared With SLND Alone in the American College of Surgeons Oncology Group Trial Z0011. J Clin Oncol.2007,25:3657-63. 32. Galimberti V, Cole BF, Zurrida $S$ et al. Axillary dissection versus no axillary dissection in patients with sentinel-node micrometastases (IBCSG 23-01): a phase 3 randomised controlled trial. Lancet Oncol.2013,14:297-305.

33. Donker M, van Tienhoven G, Straver ME et al. Radiotherapy or surgery of the axilla after a positive sentinel node in breast cancer (EORTC 10981-22023 AMAROS): a randomised, multicentre, open-label, phase 3 non-inferiority trial. Lancet Oncol.2014,15:1303-10. 


\section{Figures}

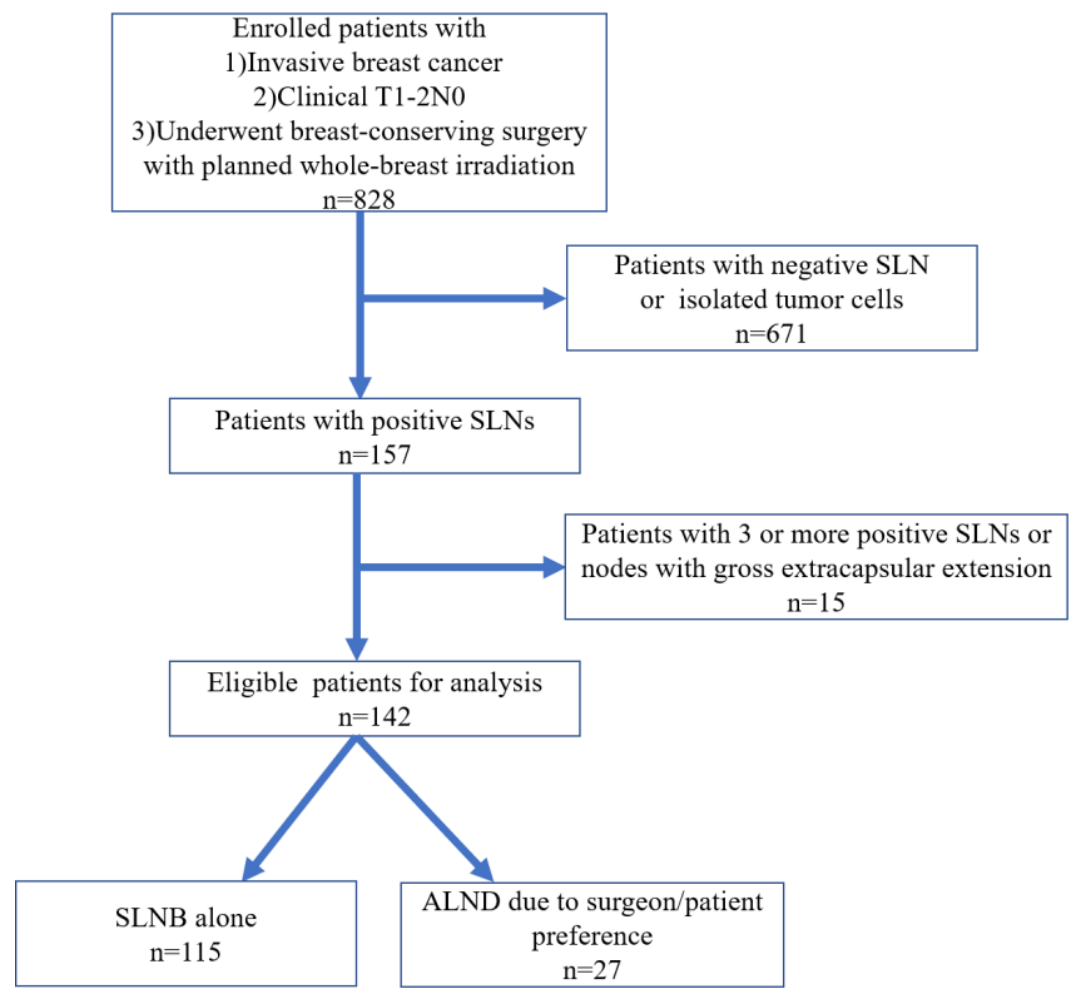

Fig 1. Flow chart of the study procedures

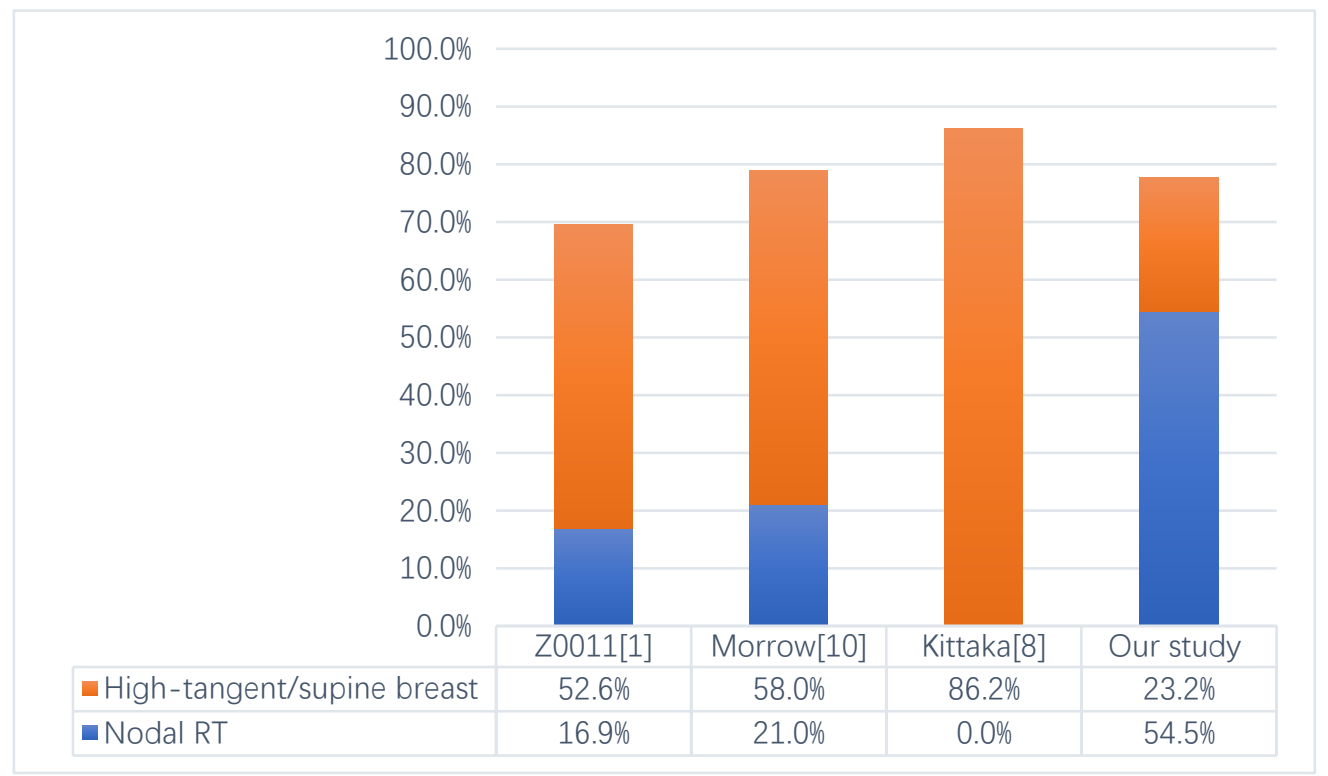

Fig 2. Radiotherapy field in the prospective studies 
Tables

Table 1. Baseline characteristics of eligible patients $(n=142)$

\begin{tabular}{llll}
\hline $\begin{array}{l}\text { Clinicopathological } \\
\text { characteristics }\end{array}$ & $\begin{array}{c}\text { Total } \\
(\mathrm{n}=142)\end{array}$ & $\begin{array}{c}\text { SLNB alone } \\
(\mathrm{n}=115)\end{array}$ & $\begin{array}{c}\text { ALND } \\
(\mathrm{n}=27)\end{array}$ \\
\hline Age (median, range) & 52 years $(26-82)$ & 52 years $(29-82)$ & 44 years $(26-69)$ \\
\hline $\begin{array}{l}\text { Age group, no. }(\%) \\
\quad \leq 50\end{array}$ & $68(47.89)$ & $51(44.35)$ & $17(62.96)$ \\
$\quad>50$ & $74(52.11)$ & $64(55.65)$ & $10(37.04)$ \\
Clinical T stage, no. $(\%)$ & & & \\
$\quad$ cT1 & $112(78.87)$ & $90(78.26)$ & $22(81.48)$ \\
$\quad$ cT2 & $30(21.13)$ & $25(21.74)$ & $5(18.52)$
\end{tabular}

Pathological T stage, no.

(\%)

pT1
pT2
Tumor histology, no. (\%)
Ductal

103 (72.54)

$81(70.43)$

22 (81.48)

39 (33.91)

34 (29.57)

$5(18.52)$

Lobular

119 (83.80)

95 (82.61)

24 (88.89)

Other

15 (10.56)

13 (11.30)

2 (7.41)

8 (5.63)

7 (6.09)

1 (3.7)

Lymphovascular

invasion, no. (\%)

Present

33 (23.24)

$26(22.61)$

7 (25.93)

Absent

68 (47.89)

$60(52.17)$

8 (29.63)

Missing

41 (28.87)

$29(25.22)$

12 (44.44)

Hormone status, no. (\%)

Positive

Negative

117 (82.39)

$92(80.0)$

25 (92.59)

25 (17.61)

23 (20.0)

2 (7.41)

HER2 status, no. (\%)

$$
\text { Negative }
$$

118 (83.10)

$96(83.48)$

22 (81.48)

Positive

Unknown

21 (14.79)

17 (14.78)

4 (14.81)

3 (2.11)

2 (1.74)

$1(3.70)$

Number of positive SLN, no. $(\%)$

$$
1
$$

2

109 (76.76)

94 (81.74)

15 (55.56)

33 (23.24)

21 (18.26)

12 (44.44)

Adjuvant chemotherapy, no. (\%)

$$
\text { Yes }
$$

No

$129(90.45)$

13 (9.15)

$102(88.70)$

13 (11.30)

27 (100.00)

$0(0.00)$

Adjuvant endocrine therapy, no. (\%)

$$
\text { Yes }
$$

115 (80.99)

92 (80.00)

23 (85.19)

No

23 (20.00)

4 (14.81) 
Radiotherapy, no. (\%)

$\begin{array}{lll}\text { Yes } & 127(89.44) & 101(87.83)\end{array}$

No $15(10.56) \quad 14(12.17) \quad 1(3.70)$

Table 2. Comparison of characteristics between patients treated with whole-breast irradiation alone, high-tangent radiotherapy and treated with nodal radiotherapy

\begin{tabular}{|c|c|c|c|}
\hline Characteristics & $\begin{array}{l}\text { Whole-breast alone } \\
(\mathrm{n}=22)\end{array}$ & $\begin{array}{l}\text { High tangent } \\
(\mathrm{n}=23)\end{array}$ & $\begin{array}{l}\text { Breast }+ \text { nodes } \\
(\mathrm{n}=54)\end{array}$ \\
\hline Age, $($ median) years & 52.5 & 55 & 49.5 \\
\hline $\begin{array}{l}\text { Pathological } \mathrm{T} \text { stage, }(\mathrm{cm} \text {; } \\
\text { mean) }\end{array}$ & 1.51 & 1.78 & 1.89 \\
\hline \multicolumn{4}{|l|}{ Hormone status, no. (\%) } \\
\hline Positive & $20(90.91)$ & $20(86.96)$ & $43(79.63)$ \\
\hline Negative & $2(9.09)$ & $3(13.04)$ & $11(20.37)$ \\
\hline \multicolumn{4}{|l|}{ HER2 status, no. (\%) } \\
\hline Negative & $20(95.24)$ & $21(91.30)$ & $42(79.25)$ \\
\hline Positive & $1(4.76)$ & $2(8.70)$ & $11(20.75)$ \\
\hline Unknown & 1 & 0 & 1 \\
\hline \multicolumn{4}{|l|}{$\begin{array}{l}\text { Lymphovascular invasion, no. } \\
(\%)\end{array}$} \\
\hline Present & $4(22.22)$ & $7(43.75)$ & $14(34.15)$ \\
\hline Absent & $14(77.78)$ & $9(56.25)$ & $27(65.85)$ \\
\hline Missing & 4 & 7 & 13 \\
\hline \multicolumn{4}{|l|}{ Number of positive SLN } \\
\hline one & $20(90.91)$ & $20(86.96)$ & $41(75.93)$ \\
\hline two & $2(9.09)$ & $3(13.04)$ & $13(24.07)$ \\
\hline
\end{tabular}

Table 3. Clinicopathological characteristics between patients in the Z0011 SLNB-alone arm and the current study

\begin{tabular}{llll}
\hline Characteristics & Eligible patients $(\mathrm{n}=142)$ & $\begin{array}{l}\text { Z0011 SLNB alone } \\
(\mathrm{n}=436)\end{array}$ & $P$ Value \\
\hline $\begin{array}{l}\text { Age, median (range), years } \\
\text { Age group, no. (\%) }\end{array}$ & $52(26-82)$ & $54(25-90)$ & - \\
$\quad$ 50 & $68(47.89)$ & $160(37.6)$ & 0.03 \\
$>50$ & $74(52.11)$ & $266(62.4)$ & \\
$\quad$ Missing & 0 & 10 & \\
Clinical T stage, no. (\%) & & & \\
$\quad$ cT1 & $112(78.87)$ & $303(70.6)$ & \\
$\quad$ cT2 & $30(21.13)$ & $126(29.4)$ & \\
$\quad$ Missing & 0 & 7 & \\
Tumor histology, no. $(\%)$ & & &
\end{tabular}


Ductal

Lobular

Other

Missing

Hormone status, no. (\%)

Positive

Negative

Missing

Lymphovascular invasion, no. $(\%)$

Present
Absent
Missing

Number of positive SLN, no. $(\%)$

$\begin{array}{ll}0-1 & 109(76.76) \\ \geq 2 & 33(23.24) \\ \text { Missing } & 0\end{array}$

Adjuvant chemotherapy,

no. $(\%)$

Yes

No/Missing

Adjuvant endocrine

therapy, no. (\%)

Yes

No/Missing

Radiotherapy, no. (\%)

Yes

No

$*_{n}=309$
33 (32.67)

$68(67.33)$

41

113 (35.2)

0.641

208 (64.8)

115

$324(78.1)$

91 (21.9)

21

$129(90.85)$

$13(9.15)$

$356(84.0)$

0.589

$36(8.5)$

$32(7.5)$

12

328 (83.7)

0.726

64 (16.3)

44

$253(58.0)<0.001$

183 (42.0)

115 (80.99)

$203(46.6)$

$<0.001$

27 (19.01)

233 (53.4)

$127(89.44)$

$277 *(89.64)$

0.947
$32 *(10.36)$

Table 4. The BCLE-SEI symptom score among the eligible patients

\begin{tabular}{llll}
\hline Symptom score & SLNB-alone $(\mathrm{n}=115)$ & ALND $(\mathrm{n}=27)$ & $P$ Value \\
\hline $0, \mathrm{n}(\%)$ & $104(90.43)$ & $22(81.48)$ & $<0.001$ \\
$2-8, \mathrm{n}(\%)$ & $11(9.57)$ & $5(18.52)$ & \\
$\geq 9, \mathrm{n}(\%)$ & 0 & 0 & \\
\hline
\end{tabular}


Table 5. Additional positive nodes after ALND in eligible patients based on the Z0011 criteria

\begin{tabular}{lll}
\hline Author & $\begin{array}{l}\text { Number of } \\
\text { patients }\end{array}$ & $\begin{array}{l}\text { Non-SLN } \\
\text { positive }\end{array}$ \\
\hline Giuliano [1] & 355 & $27.3 \%$ \\
Aigner [6] & 132 & $39 \%$ \\
Delpech [5] & 87 & $29 \%$ \\
Miao Liu [15] & 151 & $25.2 \%$ \\
Verheuvel [19] & 625 & $26 \%$ \\
Ngui [7] & 22 & $27.3 \%$ \\
Present study & 27 & $25.94 \%$ \\
\hline
\end{tabular}




\section{Figures}

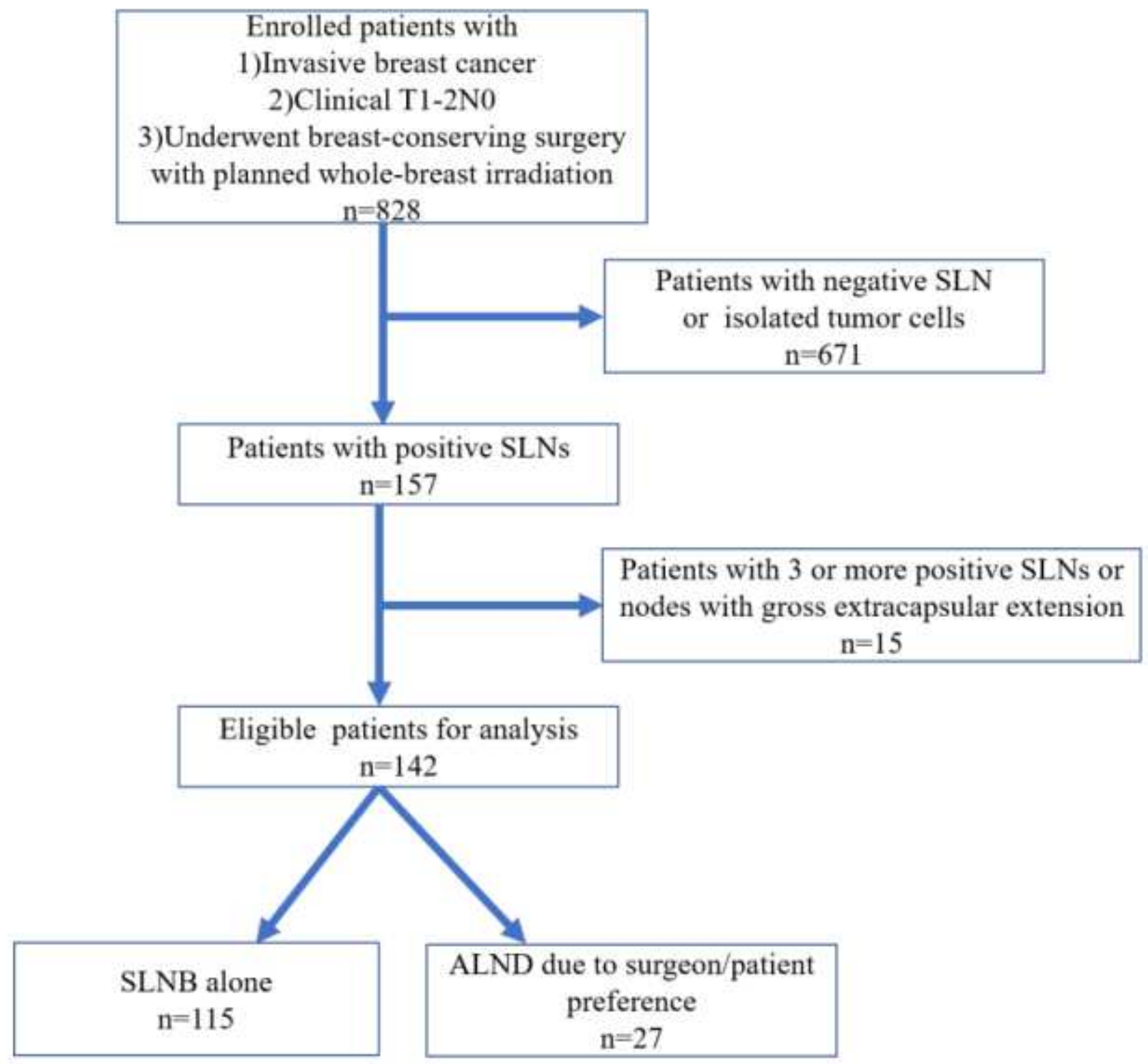

Figure 1

Flow chart of the study procedures 


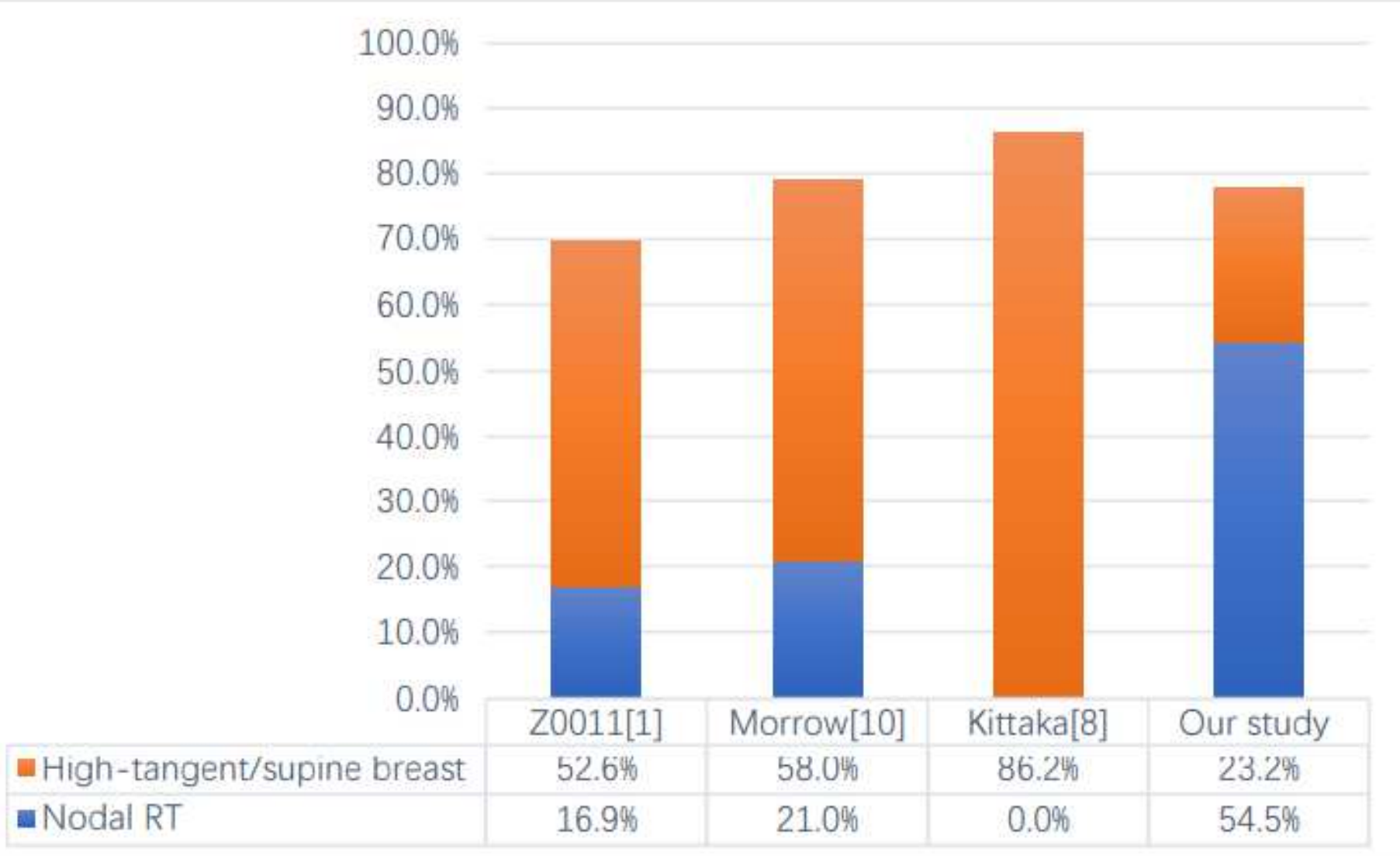

Figure 2

Radiotherapy field in the prospective studies 